\title{
Stability Data Xbee S2b Zigbee Communication on Arduino Based Sumo Robot
}

\author{
I Gusti Made Ngurah Desnanjaya ${ }^{1 *}$, I Made Aditya Nugraha ${ }^{2 *}$, I Wayan Dani Pranata ${ }^{3}$, Wawan Harianto \\ 1,3,4 Computer Systems Study, STMIK STIKOM Indonesia \\ ${ }^{2}$ Fisheries Mechanization, Marine and Fisheries Polytechnic of Kupang \\ Email: ${ }^{1}$ ngurah.desnanjaya@ stiki-indonesia.ac.id, ${ }^{2}$ made.nugraha@kkp.go.id, ${ }^{3}$ pranatadani92@gmail.com, \\ ${ }^{4}$ wawanhariantoxxxx@gmail.com \\ *Corresponding Author
}

\begin{abstract}
Wireless technology is a solution to reach hardto-reach places using cable technology. The use of wireless technology can reach these areas without using copper or cables in the data transmission process. Communication technology using wireless is one solution to connect the control system to support the creation and achievement of the goals of the 4.0 revolution so that control and monitoring become easier. With the convenience provided by wireless technology such as connecting various types of system devices between other systems. One type of wireless technology is WPAN, especially on the ZigBee system from Digi International with the IEEE 802.15.4 protocol standard with low power consumption and working at a frequency of $2.4 \mathrm{GHz}$. Utilization of the communication system from the ZigBee protocol can control according to the range capabilities of the ZigBee system. ZigBee's ability and use of communication can perform communication control robot sumobot (slave robot) and remote control (robot leader) as an introduction to command data to carry out movements on the slave robot in accordance with the orders from the robot leader After trying XBEE to send data at a certain distance without experiencing data loss, the best distance is obtained, namely $1-100$ meters with the firmware coordinator and router settings. Data delays can be minimized with a transfer rate of $3 \mathrm{kbps}$ in the data transfer rate.
\end{abstract}

Keywords-ZigBee, XBEE Communication, Sumobot, Robot Sumo, WPAN

\section{INTRODUCTION}

In the era of the industrial revolution 4.0, it refers to very rapid technological advances. Wireless technology or what is often called a wireless sensor network is a technology that is used for monitoring or monitoring. This development in the European region which has used various types of technological innovations has greatly helped humans in various fields, be it defense, health, agriculture, fisheries, clothing, food and others [1][2][3][4].

As we know, Indonesia is entering the era of the industrial revolution 4.0. The nation's children have started to carry out various types of innovations to help human work itself [5] [6]. In the current technological era, the use of cables as a conductor for current signals is very widely used. If all are connected using a cable, this will inhibit various activities and large costs [7][8][9]. To overcome this, you can use wireless technology / wireless [10][11].

Wireless technology is able to connect the device with other connected devices [12][13][14][15]. At this time, wireless communication is very much in demand, such as the WPAN type or for medium or medium networks. The ability of this wireless is reliable in the efficiency of time and installation costs. One of the most widely used WPAN technologies for communication between devices today is the ZigBee model from Digi International [16][17][18][19]. This ZigBee is standardized with IEEE 802.15.4 with low power consumption for short distances and works-on a frequency of $2.4 \mathrm{GHz}[20][21][22][23][24]$.

Research conducted by researchers is supported by the results of research conducted by previous people regarding the concept of testing communication on XBEE (Zigbee). From previous research, the researchers tested the work of XBEE and its abilities and tested it by experimenting with several important measured values [25][26]. Analysis of previous research performance can be continued and developed by communicating in a remote-controlled sumo robot system[27][28][29]. The concept that is built is remote, which will send a signal in the form of a command that is forwarded to the sumo robot. This information signal can be synchronized with execution with the command given [21].

The STMIK STIKOM Indonesia agency in the Control System Laboratory section has a sumobot robot but still uses the bluetooth communication type. Researchers conducted a research experiment by trying to design a remote control to become a robot leader as a transmitter and a sumobot or slave robot as a data receiver [15] [30][31]. The experiment that was carried out was trying to implement the peer to peer communication module XBEE / wireless on each device.

A.

Comparison with other studies

Researchers conducted this research because the results of previous studies could only reach a distance of 24 meters and experienced interference [32]. This study did not explain the number of repetitions performed. This initial limitation of the Robo Sumo was then fixed by using good quality and transfer distance to get a quick response to the controller for data execution. This data delay can be minimized by using XBEE S2B which has a possible maximum transfer distance of 500 meters [23]. The results of this study, the researchers developed by testing the XBEE S2B to transmit data without losing data with good speed quality and minimizing packet loss.

Researchers conducted this research because the results of previous studies could only reach a distance of 24 meters and experienced interference [32]. This study did not explain the number of repetitions performed. This initial limitation of the Robo Sumo was then fixed by using good quality and transfer distance to get a fast response to the controller for data 
execution. This data delay can be minimized by using XBEE S2B which has a possible maximum transfer distance of 500 meters [23]. The results of this study, the researchers developed by testing the XBEE S2B to transmit data without losing data with good speed quality and minimizing data packet loss.

\section{B. An explanation of the initial sumo robot}

STMIK STIKOM Indonesia has a sumo robot using Bluetooth communication with connectivity to a smartphonebased on an app controller to run the system on the robot. However, the robot experienced many problems such as frequent data delays or data loss using the HC-6 Bluetooth module. from that, the researcher tried an experiment to develop it so that data sent from the remote get a fast response When getting commands via the remote controller with the ability of the XBEE S2B itself, it is able to support it without burdening the IC controller because XBEE has its own delivery system or firmware so that the respond coordinator and the router can be more efficient so that data loss such as Bluetooth HC-6 can be minimized.

\section{DESIGN METHODS}

Researchers will explain the system to be built on the sumo robot with several important values to test the functioning of the robot properly.

In this case, the researcher replaced the existing system in the initial sumo robot with XBEE due to several factors as follows:

1. There is often a delay in receiving data

2. Researchers want to develop a better XBEE response on Robo Sumo

3. The use of XBEE in wireless technology in the industrial era 4.0. This development is for further research.

4. The advantages of XBEE in digital communication, where it is possible to focus data on a channel so that at a minimum distance the communication can exchange without obstacles within a certain distance, as well as the stability of XBEE.

A comparison of the use of XBEE with Bluetooth in the initial system does not work optimally, such as delays that affect the response of Robo Sumo when compared to XBEE which has good enough firmware to support communication over medium distances. XBEE itself has an antenna extender to amplify the signal.

\subsection{Remote Controller (Leader)}

The remote controller will be in charge of sending data in the form of information with the aim of being an order to synchronize command data and execution according to the slave robot (robot sumo).

TABLE I. REMOTE CONTROL COMPONENTS

\begin{tabular}{lc}
\hline No & Components \\
\hline 1 & Arduino nano \\
2 & Cables \\
3 & Joystick module \\
4 & Battery 3.7 V \\
5 & Switch \\
6 & LED \\
7 & XBEE (Transiver) \\
\hline
\end{tabular}

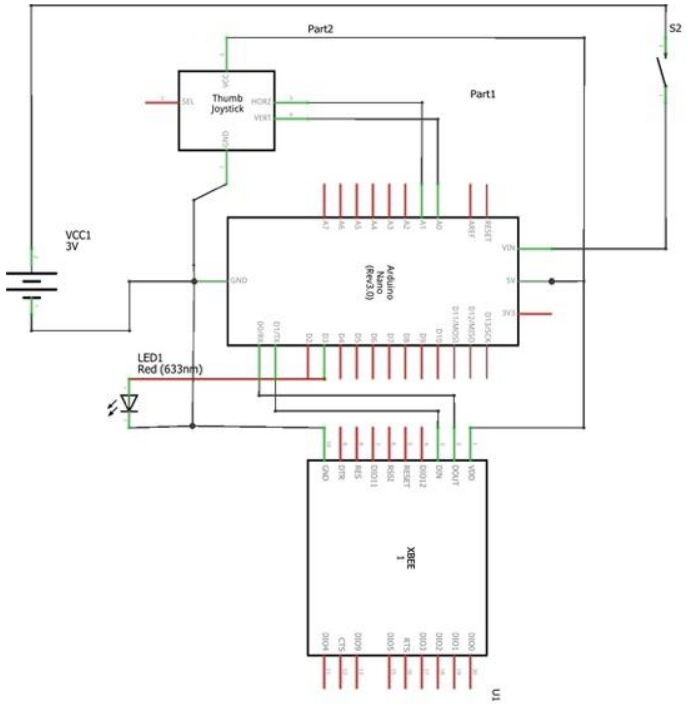

Fig. 1. Remote Control Schematic

The components in Table I will be a remote control system to control all activities that will be carried out by the sumo robot. These components will be built in the form of a remote control system. The schematic form and block diagram of the system being built can be seen in Figures 1 and 2 .

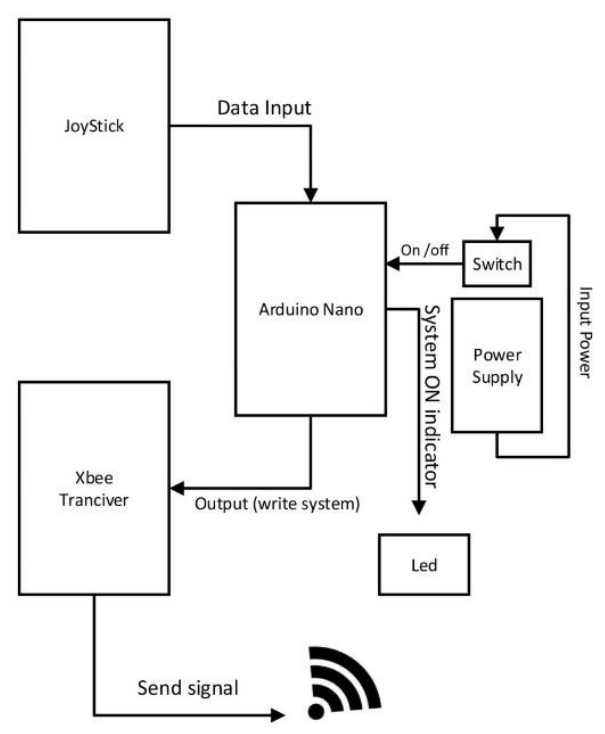

Fig. 2. Remote Controller Block Diagram

\subsection{Sumo Robot (Slave)}

The Sumo robot is a robot that receives signals from the robot leader and executes the data. Sumo robot transfer according to the command sent from the remote controller or leader. Table II below is the components of the sumo robot.

TABLE II. SUMO ROBOT COMPONENTS

\begin{tabular}{cc}
\hline No & Components \\
\hline 1 & Arduino Uno \\
2 & Cables \\
3 & $2 \times$ Motor DC \\
4 & Battery 12 V \\
5 & Switch \\
6 & LED \\
7 & 2 x Motor Driver \\
8 & XBEE (reciver) \\
\hline
\end{tabular}


The components above will be designed as a system that can be controlled by a remote controller. From these components, you will get a system that becomes one unit. The schematic form and block diagram of the sumo robot system can be seen in Figures 3 and 4.

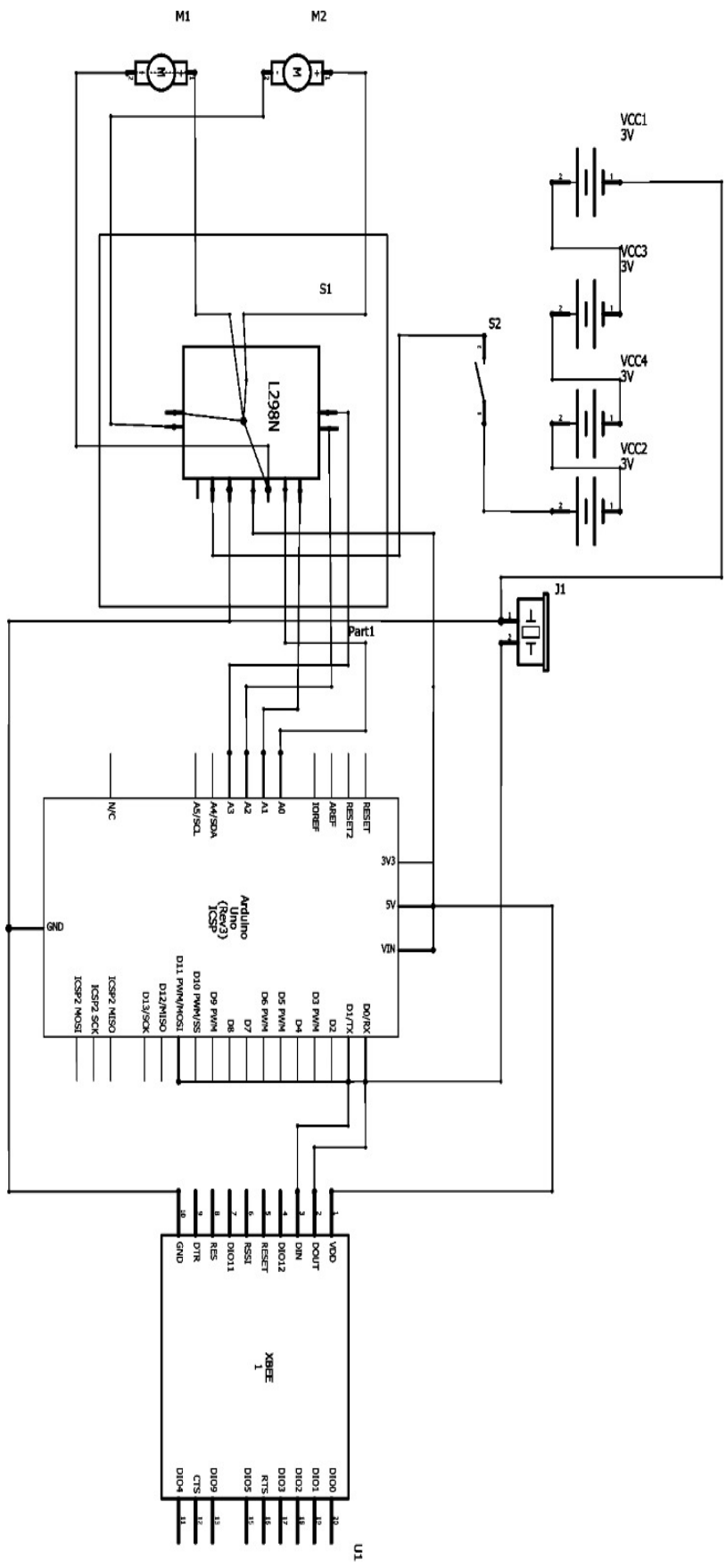

Fig. 3. Sumo Robot Schematic

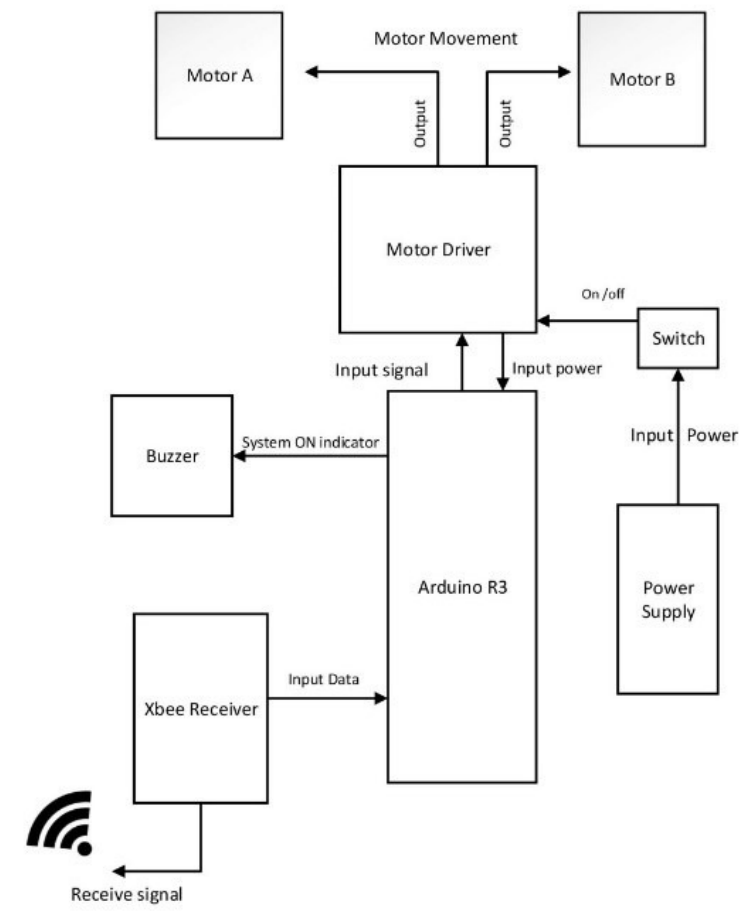

Fig. 4. Sumo Robot Block Diagram

\subsection{XBEE Configuration}

To be able to communicate between the robot leader and the slave robot, it is necessary to exchange information or it can be called communication. The purpose of this communication, namely the remote control as a robot leader will send commands then forward it to XBEE (transceiver) to be sent to XBEE (receiver) and then forwarded back to the slave robot microcontroller to execute data converted into movement and displacement of the robot in accordance with a signal received.

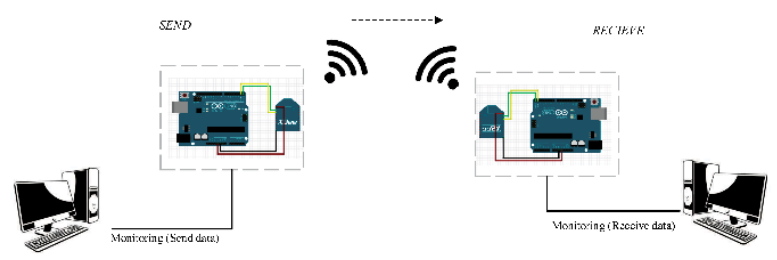

Fig. 5. XBEE Configuration Communication Scheme

The configuration on each module is in accordance with the scheme in Figure 5. From the picture above, it can be seen that the XBEE coordinator is the transceiver or the one in charge of sending data and the XBEE router is the receiver or the receiver of the data signal. This arrangement aims to exchange data so that data transmission or communication can occur between the XBEE coordinator and the router. From this communication, it will be used to send data from the remote microcontroller to the robot microcontroller so that the robot can be controlled according to the execution signal sent.

\subsection{System Communication Flowchart}

The system communication flowchart is a part that explains the communication system from the remote control with the sumo robot how data processing occurs in the system 
life cycle. From the flowchart flow, we can find out how the incoming data is executed when a signal is sent from the remote controller to the Sumo robot. Communication with the data flow concept is described in the form of symbols as a representation of how the data is processed by the system. Figure 6 below is the form of a system flowchart that is built.

\subsection{System Testing Methods}

In testing the design that has been built, a capability test will be carried out which aims to test the limitations of the system that has been designed. Inline, it can be drawn from this test that the function from the transceiver to the receiver is in accordance with the desired execution. Here are the important points tested:

- Testing XBEE communication in communications rarely 100 meters range.

- Testing the sumobot robot to make a full round of hands

- Testing the sumobot robot to do a full left turn
- Testing the sumobot robot for forwarding movement

- Sumobot robot testing to perform the reverse movement

- Testing the sumobot robot to make a right turn forward in a circle

- Testing the sumobot robot to turn left forward in a circle

- Testing the sumobot robot to turn right backward in a circle

- Testing the sumobot robot to turn left backward in a circle

From these tests, the researcher obtained the results of controlling the robot with a remote control in accordance with the signal sent and received by the slave robot. The robot can move according to the instructions received from the robot leader or remote control.

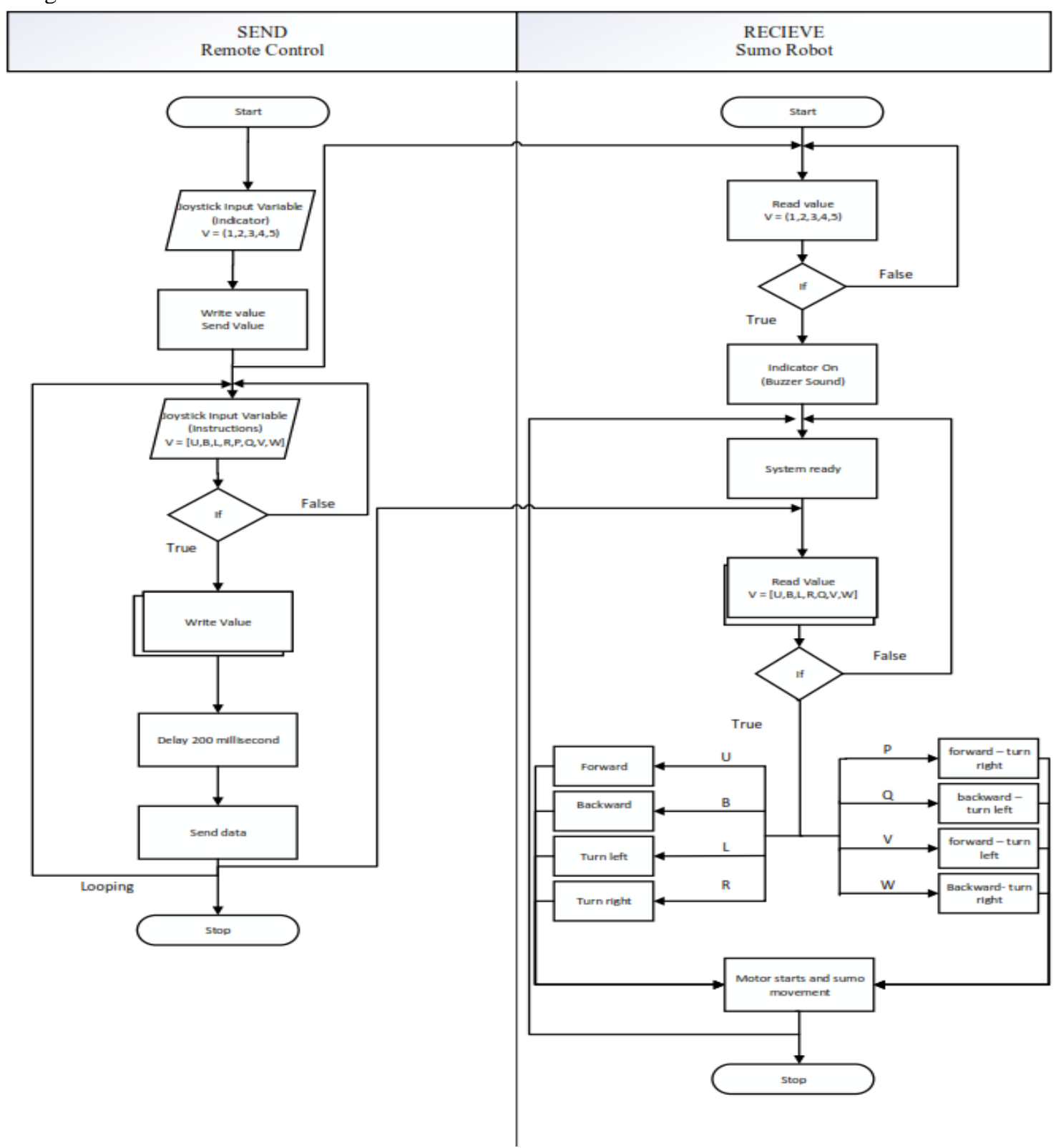

Fig. 6. Communication System Flowchart 


\section{IMPLEMENTATION OF RESULTS}

Testing the best ability of XBEE at a distance of 100 meters. This is better than the research conducted by Hartawan where the XBEE ability in open space is estimated to reach 600 meters.

Researchers try to find the best scale to test the system so that the data received is not too late for robot communication. After being analyzed with the sumo robot signal communication it was decided to test up to 100 meters at the line of sight or without obstruction. The main objective is to get the best communication distance for the coordinator and router to be able to communicate with the maximum capability of the module.

The XBEE ability test was conducted at Jln. Tukad Citarum, Puri Klod Lake, South Denpasar, Bali. The test was carried out 2 times, namely testing using XTCU software on XBEE and testing with a microcontroller. Figure 7 is the XBEE test location.

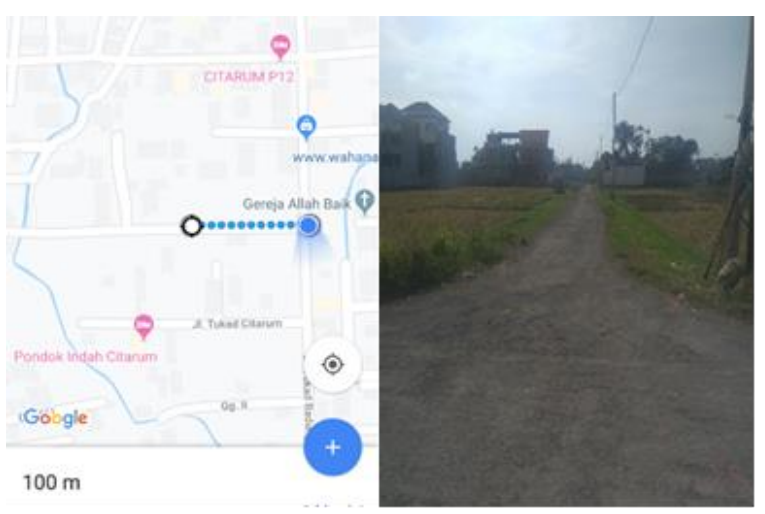

Fig. 7. XBEE Test Locations

\subsection{Testing Using XTCU}

In testing the xtcu software was used. The test was carried out using 2 methods, namely the range test and throughput testing. Tables 3 and 4 are the results of the XBEE range test data and the results of the XBEE coordinator and router test data.
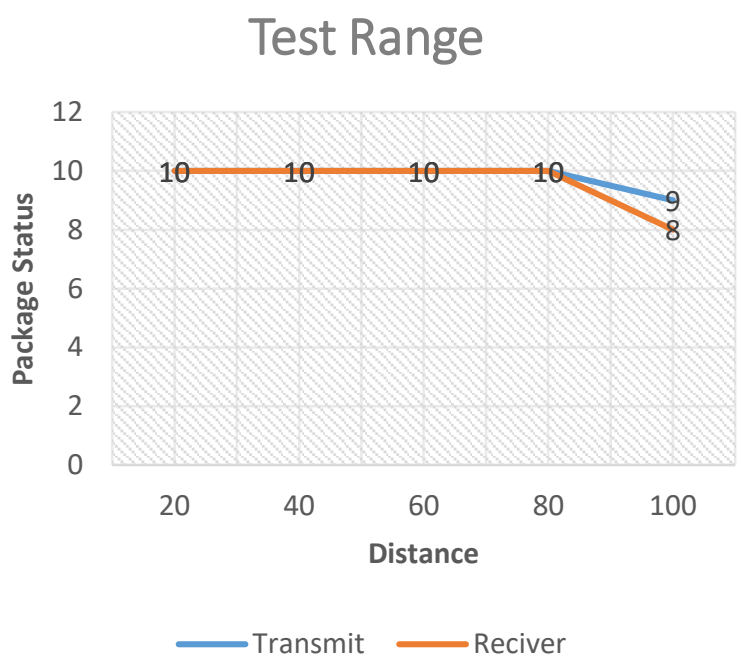

Fig. 8. XBEE Range Test Data Graph
When sending data with signal quality as in Table III below, the results are that the packet is sent properly on the line of sight when transferring data with a distance of 100 meters. The transmitter failed to send 1 packet and the receiver failed to receive 1 packet with each packet load of 84 bytes.

TABLE III. TEST DATA RESULTS TEST RANGE XBEE

\begin{tabular}{ccccccc}
\hline \multirow{2}{*}{$\begin{array}{c}\text { Distance } \\
\text { (Meter) }\end{array}$} & \multicolumn{2}{c}{ Coordinator } & \multicolumn{2}{c}{ Router } & \multicolumn{2}{c}{ Status } \\
\hline 20 & 3 & $-61 \mathrm{dBm}$ & 2 & $-77 \mathrm{dBm}$ & 10 & 10 \\
40 & 3 & $-60 \mathrm{dBm}$ & 3 & $-65 \mathrm{dBm}$ & 10 & 10 \\
60 & 3 & $-60 \mathrm{dBm}$ & 2 & $-68 \mathrm{dBm}$ & 10 & 10 \\
80 & 2 & $-68 \mathrm{dBm}$ & 2 & $-74 \mathrm{dBm}$ & 10 & 10 \\
100 & 2 & $-72 \mathrm{dBm}$ & 2 & $-74 \mathrm{dBm}$ & 9 & 8 \\
\hline
\end{tabular}

\section{- Throughtput}

Throughput testing is to test the speed of packet transfer capability on XBEE. Table IV is the test results from the throughput test.

\begin{tabular}{ccccc}
\multicolumn{5}{c}{ TABLE IV. RESULTS OF TESTING DATA FOR XBEE } \\
COORDINATOR AND ROUTER \\
\hline Distance & \multicolumn{2}{c}{ Speed transfer } & $\begin{array}{c}\text { Transmit paket } \\
\text { /10s }\end{array}$ & receiver \\
(Meter) & $\begin{array}{c}\text { Average } \\
(\mathrm{Kbps})\end{array}$ & $\begin{array}{c}\text { Max } \\
(\mathrm{Kbps})\end{array}$ & & \\
\hline 20 & 3.1 & 3.11 & 16 & 16 \\
40 & 3.09 & 3.13 & 16 & 16 \\
60 & 3.1 & 3.12 & 16 & 16 \\
80 & 3.1 & 3.12 & 16 & 16 \\
100 & 3.08 & 3.12 & 16 & 16 \\
\hline
\end{tabular}

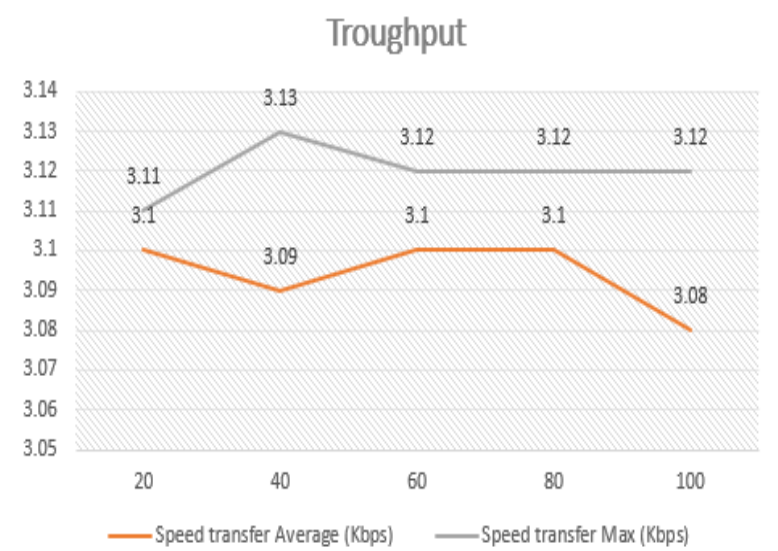

Fig. 9. Graph of XBEE Coordinator and Router Test Data

The above test is performed to transfer 1000 bytes of data with a transfer time of 5 seconds. The results of the throughput test above can prove that the distance is getting wider the speed or the speed of data transfer decreases, but at a distance of 100 meters with a line of sight, it is still good to use it for data transfer.

\subsection{XBEE testing using Arduino Uno}

Testing using the Arduino Uno R3 aims to test data packet transfers. Testing is done by sending a data packet, "hello world" and checking for missing part of the character with a test distance of 100 meters. Each data packet delivery has a 
delay of 2 seconds. The test was carried out 3 times with the same location. Table $\mathrm{V}$ is the data from the results of the tests carried out.

Arduino test results as a coordinator and router within 100 meters are still good at transferring data. No packet length is lost. It can be said that in a distance of 20 meters to 100 meters implemented on the Arduino it can be said to be good because the data packet "hello world" sent has no missing characters.

TABLE V. TESTING DATA FOR XBEE ARDUINO COORDINATOR AND ROUTER

\begin{tabular}{cccc}
\hline $\begin{array}{c}\text { Distance } \\
\text { (Meter) }\end{array}$ & \multicolumn{2}{c}{ Arduino (Paket) } & Status \\
\hline 20 & 10 & Reciver & \\
40 & 10 & 10 & Success \\
60 & 10 & 10 & Success \\
80 & 10 & 10 & Success \\
100 & 10 & 10 & Success \\
\hline
\end{tabular}

3.3 Remote Controller Configuration Data

Researchers designed a simple remote control using an analog joystick input with an Arduino nano microcontroller and a transmitter in the form of an XBEE coordinator module. The remote serves as the robot leader (instruction center) for the slave robot (instruction execution).

To control the movement, it is necessary to determine the range division for the joystick direction instruction as the command center for the sumo robot. The division of the direction is divided into the ADC value of the joystick module according to the consumption of the direction taken. Figure 10 is a form of joystick direction configuration designed by researchers.

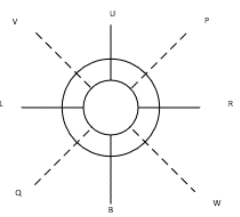

Fig. 10. Sketch Map Module Joystick

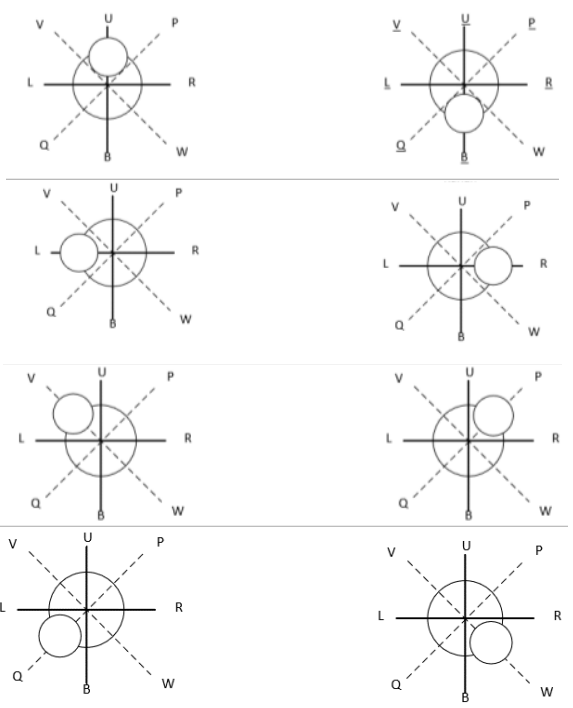

Fig. 11. The Appraisal Part of The Joystick Movement

Determining the mapping direction will make it easier for the instructions to be sent by the XBEE coordinator to the
$\mathrm{XBEE}$ receiver as control instructions for the sumobot robot. $\mathrm{ADC}$ division is very important so there is no collision of the same value or value. ADC x-axis 0 - 255 and $y$-axis from 0 255. Each symbol is based on the $\mathrm{x}$ and $\mathrm{y}$-axis values which are divided into 8 parts as a measure of the value sent. This description can be seen in Figure 11. This data is useful for explaining the communication data received by Robo Sumo sent by the controller.

\subsection{Remote and Robot System Assembly}

Assembling remote systems and robots aims to tidy up the various placement of components so as not to interfere with the execution process when the robot is alive, in other words when there is communication between the remote and the robot.

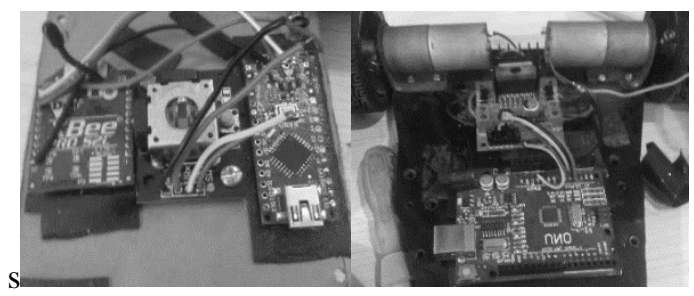

Fig. 12. Robot Assembly Process

Many things must be taken into account in placing the position of the system. Good placement of several components can provide good performance and reduce short or short.

\subsection{Robot Communication Testing}

Researchers conducted robot testing aimed at whether the robot was able to run properly and in accordance with commands from the remote control. From this test, the researchers obtained the data in Table VI.

TABLE VI. RESULTS OF TESTING MOVEMENT ON THE TRACK

\begin{tabular}{|c|c|c|c|}
\hline No & Remote Control & Testing & Description \\
\hline 1 & Signal U / Forward & $\begin{array}{l}\text { Move forward / } \\
\text { Forward }\end{array}$ & Success \\
\hline 2 & Signal B / Backward & Move Back / Reverse & Success \\
\hline 3 & Signal R / Right Turn & Move Turn to right & Success \\
\hline 4 & Signal L / Left Turn & $\begin{array}{l}\text { Move around to the } \\
\text { Left }\end{array}$ & Success \\
\hline 5 & $\begin{array}{l}\text { Signal P / Move Circle } \\
\text { Right - Forward }\end{array}$ & $\begin{array}{l}\text { Circular move forward } \\
\text { by turning right }\end{array}$ & Success \\
\hline 6 & $\begin{array}{l}\text { Signal V / Move Circle } \\
\text { Left - Forward }\end{array}$ & $\begin{array}{l}\text { Move forward circular } \\
\text { with left turn }\end{array}$ & Success \\
\hline 7 & $\begin{array}{l}\text { Signal W / Move } \\
\text { Circular left - backward }\end{array}$ & $\begin{array}{l}\text { Circle move backwards } \\
\text { by turning right }\end{array}$ & Success \\
\hline 8 & $\begin{array}{l}\text { Signal Q / Move left } \\
\text { circle - Backward }\end{array}$ & $\begin{array}{l}\text { Move circle backwards } \\
\text { by turning left }\end{array}$ & Success \\
\hline
\end{tabular}

\subsection{Testing Description}

Figure 13 shows the experiments conducted by researchers in system testers. The following is a description of the symbols in the image above:

$\mathrm{U}$ : Move forward orders.

B : Movement order backwards.

L : Command turn left/turn left, by fighting at the last stop point.

$\mathrm{R}$ : Command turn right / turn right, relying on the last stop point. 
$\mathrm{P} \quad$ : Command circular rotation right forward / half-right turn and not resting on the endpoint of stop.

V : Command circular left forward / half turn left and not resting on the last stop point.

W : Command circular right reverse/half reverse right and not resting on the last stop point.

Q : Command circular left reverse/half reverse left and not resting on the last stop point.

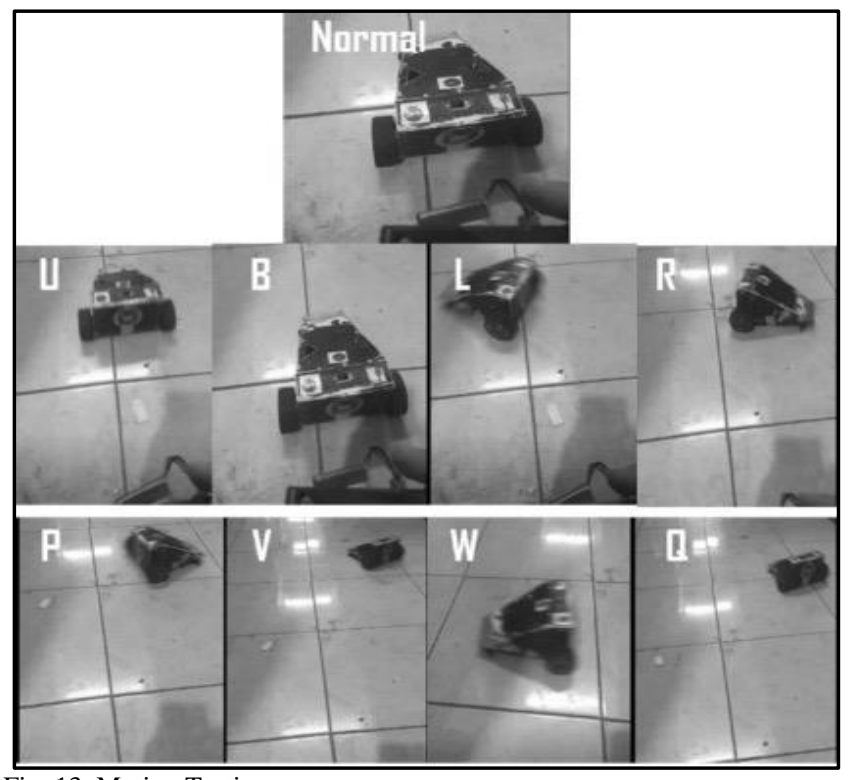

Fig. 13. Motion Testing

From the experimental results above, it shows that the slave robot and leader robot are in sync. The command sent by the robot leader is in accordance with the value execution carried out by the slave robot and a good signal range for the XBEE module as a communication medium for robots such as the sumobot robot. This is because this XBEE module has low power consumption or low voltage so it can save power consumption for the system.

\section{CONCLUSION}

From the results of research and experiments conducted by researchers to obtain stability data from XBEE, it can be seen that the Sumobot robot receives fast data with good stability. After trying XBEE to send data at a certain distance without experiencing data loss, the best distance is obtained, namely 1 - 100 meters with the firmware coordinator and router settings.

Implementation of the system can work well in covering communications that occur in remote systems and sumo robots. Data delays can be minimized with a transfer rate of 3 kbps in the data transfer rate. Matching communication criteria with what the system needs must be adjusted so that it can meet the objectives of the system being built.

In this study, the researcher only wanted to achieve the goal of communicating the robot leader and slave to become one system. If the next researcher wants to develop, the researcher gives a little advice in its development. Such as the addition of supporting features to help and monitor the transmission system as well as a more elegant indicator in its development and development of a system on a single board that supports the shape and pattern of the robot itself. And one more thing, the development with XBEE communication can be developed again in various controlled devices to fulfill the integrity of control systems such as RC boats, cars, networks, control, and so on.

\section{REFERENCES}

[1] Y. Zakaria and K. Michael, "An Integrated Cloud-Based Wireless Sensor Network for Monitoring Industrial Wastewater Discharged into Water Sources," Wirel. Sens. Netw., 2017.

[2] S. Pirbhulal et al., "A novel secure IoT-based smart home automation system using a wireless sensor network," Sensors (Switzerland), 2017.

[3] H. C. Lee and H. H. Lin, "Design and Evaluation of an Open-Source Wireless Mesh Networking Module for Environmental Monitoring," IEEE Sens. J., 2016.

[4] X. Tian et al., "Wireless body sensor networks based on metamaterial textiles," Nat. Electron., 2019.

[5] R. Gravina, P. Alinia, H. Ghasemzadeh, and G. Fortino, "Multi-sensor fusion in body sensor networks: State-of-the-art and research challenges," Inf. Fusion, 2017.

[6] M. Bor and U. Roedig, "LoRa transmission parameter selection," in Proceedings - 2017 13th International Conference on Distributed Computing in Sensor Systems, DCOSS 2017, 2018.

[7] A. Cama-Pinto, G. Piñeres-Espitia, J. Caicedo-Ortiz, E. RamírezCerpa, L. Betancur-Agudelo, and F. Gómez-Mula, "Received strength signal intensity performance analysis in wireless sensor network using Arduino platform and XBee wireless modules," Int. J. Distrib. Sens. Networks, 2017.

[8] N. Ahmad, A. Hussain, I. Ullah, and B. H. Zaidi, "IOT based wireless sensor network for precision agriculture," in iEECON 2019 - 7th International Electrical Engineering Congress, Proceedings, 2019.

[9] I. N. B. Hartawan, P. P. Santika, I. B. A. I. Iswara, and I. G. M. N. Desnanjaya, "Effect of electromagnetic wave interference against computer network quality of service," in Journal of Physics: Conference Series, 2020.

[10] Q. N. Cao, C. Liu, and K. Y. Meng, "Design of oilfield wireless monitoring system based on ZigBee and WiFi," Xi' an Shiyou Daxue Xuebao (Ziran Kexue Ban)/Journal Xi'an Shiyou Univ. Nat. Sci. Ed., 2015.

[11] R. P. Narayanan, T. V. Sarath, and V. V. Vineeth, "Survey on Motes Used in Wireless Sensor Networks: Performance \&amp; Parametric Analysis," Wirel. Sens. Netw., 2016.

[12] D. J. Bora, N. Kumar, and R. Dutta, "Implementation of wireless MEMS sensor network for detection of gait events," IET Wirel. Sens. Syst., 2019.

[13] I. G. M. N. Desnanjaya, I. G. I. Sudipa, and I. W. D. Pranata, "Performance Analysis Of Balinese Kulkul Beats Information System Based on Website and Android Using ISO 9126," Proceeding Electr. Eng. Comput. Sci. Informatics, 2020.

[14] Y. Li, Z. Chi, X. Liu, and T. Zhu, "Passive-ZigBee: Enabling zigbee communication in IoT networks with $1000 \mathrm{X}+$ less power consumption," in SenSys 2018 - Proceedings of the 16th Conference on Embedded Networked Sensor Systems, 2018.

[15] V. Varshney and M. Tiwari, "Realization of an FIR filter using ATMEGA32 microcontroller," in 2017 International Conference on Emerging Trends in Computing and Communication Technologies, ICETCCT 2017, 2018.

[16] D. Y. Kim, S. Kim, H. Hassan, and J. H. Park, "Adaptive data rate control in low power wide area networks for long range IoT services," J. Comput. Sci., 2017.

[17] X. Peng, J. Yin, P. I. Mak, W. H. Yu, and R. P. Martins, "A 2.4-GHz ZigBee Transmitter Using a Function-Reuse Class-F DCO-PA and an ADPLL Achieving 22.6\% (14.5\%) System Efficiency at 6-dBm (0$\mathrm{dBm})$ Pout," IEEE J. Solid-State Circuits, 2017.

[18] J. Tosi, F. Taffoni, M. Santacatterina, R. Sannino, and D. Formica, "Throughput Analysis of BLE Sensor Network for Motion Tracking of Human Movements," IEEE Sens. J., 2019.

[19] A. Zourmand, A. L. Kun Hing, C. Wai Hung, and M. Abdulrehman, "Internet of Things (IoT) using LoRa technology," in 2019 IEEE International Conference on Automatic Control and Intelligent Systems, I2CACIS 2019 - Proceedings, 2019.

[20] M. A. Moridi, Y. Kawamura, M. Sharifzadeh, E. K. Chanda, M. Wagner, and H. Okawa, "Performance analysis of ZigBee network topologies for underground space monitoring and communication systems," Tunn. Undergr. Sp. Technol., 2018.

[21] I. G. M. N. Desnanjaya and I. G. I. Sudipa, "The Control System of Kulkul Bali Based on Microcontroller," IEEE, pp. 245-251, 2019. 


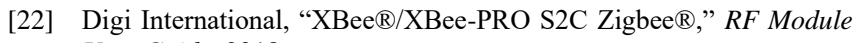
User Guide. 2018

[23] V. Deep and T. Elarabi, "Efficient IEEE 802.15.4 ZigBee standard hardware design for IoT applications," in Proceedings - International Conference on Signals and Systems, ICSigSys 2017, 2017.

[24] K. V. De Oliveira, H. M. Esgalha Castelli, S. Jose Montebeller, and T. G. Prado Avancini, "Wireless Sensor Network for Smart Agriculture using ZigBee Protocol," in 2017 IEEE 1st Summer School on Smart Cities, S3C 2017 - Proceedings, 2018.

[25] R. A. Salam, M. R. F. Islamy, M. M. Munir, E. Yuliza, Khairurrijal, and M. Irsyam, "Design and implementation of wireless sensor network on Ground movement Detection System," in Proceeding 2015 International Conference on Computer, Control, Informatics and Its Applications: Emerging Trends in the Era of Internet of Things, IC3INA 2015, 2016.

[26] A. Hussein, A. Diaz-Alvarez, J. M. Armingol, and C. OlaverriMonreal, "3DCoAutoSim: Simulator for Cooperative ADAS and Automated Vehicles," in IEEE Conference on Intelligent Transportation Systems, Proceedings, ITSC, 2018.

[27] C. R. C. Torrico, A. B. Leal, and A. T. Y. Watanabe, "Modeling and Supervisory Control of Mobile Robots: A Case of a Sumo Robot," IFAC-PapersOnLine, 2016.

[28] I. Borcoși, "the Control of a Sumo Robot.," Ann. Constantin Brancusi Univ. Targu Jiu-Letters Soc. Sci. Ser., 2017.

[29] M. Diab et al., "An Ontology for Failure Interpretation in Automated Planning and Execution," in Advances in Intelligent Systems and Computing, 2020.

[30] S.-C. Kang, W.-T. Chang, K.-Y. Gu, and H.-L. Chi, Robot Development Using Microsoft Robotics Developer Studio. 2016.

[31] I. G. M. N. Desnanjaya, I. B. A. I. Iswara, A. A. G. Ekayana, P. P. Santika, and I. N. B. Hartawan, "Automatic high speed photography based microcontroller," in Journal of Physics: Conference Series, 2020.

[32] V. Goel et al., "Design of smartphone controlled robot using bluetooth," in Lecture Notes in Electrical Engineering, 2019. 